\title{
Durabilité Des Ressources Halieutiques Au Maroc : Cas De Mehdia
}

\section{Ibtissam Motib,}

Université Ibn Tofail, Kenitra, Faculté des lettres et des sciences humaines Département de Géographie, Laboratoire Environnement, Développement et Gestion de l'espace

Doi:10.19044/esj.2020.v16n12p204 URL:http://dx.doi.org/10.19044/esj.2020.v16n12p204

\section{Résumé}

La durabilité des ressources halieutiques est un axe stratégique de la politique de développement du secteur de la pêche maritime au Maroc. La gestion rationnelle de ces ressources et son exploitation durable constituent la base pour assurer la protection et la pérennité des espèces pour les générations actuelles et futures. Au vu de la gravité des conséquences des multiples pressions qui pèsent sur la pérennité des stocks halieutiques, et sur l'écosystème marin, et aussi sur la situation socio-économique des opérateurs de la pêche, notamment à Mehdia, le souci et la responsabilité nationale visà-vis du patrimoine halieutique interpelle les pouvoirs publiques au Maroc, à mieux gérer, et conserver de manière durable les ressources halieutiques à cette zone qui ne profité que peu de la rente de sa situation stratégique. Dans cet article, on se propose d'éclairer le lecteur d'une part, sur l'état des ressources halieutiques à Mehdia, et d'autre part de démontrer le rôle de la stratégie de durabilité qui est conçue comme un processus participatif et exploratoire d'amélioration de la qualité et la quantité des ressources halieutiques au niveau de la zone d'étude.

Mots clés: Maroc, Mehdia, Ressources halieutiques, Pêche maritime, Durabilité. 


\title{
Sustainability of Fishery Resources in Morocco: The Case of Mehdia
}

\begin{abstract}
Ibtissam Motib,
Université Ibn Tofail, Kenitra, Faculté des lettres et des sciences humaines Département de Géographie, Laboratoire Environnement, Développement et Gestion de l'espace
\end{abstract}

\begin{abstract}
The sustainability of fishery resources is a strategic axis of the development policy for the maritime fishing sector in Morocco. The rational management of these resources and its sustainable exploitation form the basis for ensuring the protection and sustainability of species for present and future generations. In view of the seriousness of the multiple pressures on the sustainability of fish stocks, and on the marine ecosystem, especially in Mehdia, the concern and national responsibility vis-à-vis the fisheries heritage challenges the public authorities in Morocco, to better manage fishery resources in this area which benefits little from the rent from its strategic location. In this article, we propose to enlighten the reader on the one hand, on the state of fishery resources in Mehdia, and on the other hand to demonstrate the role of the sustainability strategy which is conceived as a participatory and exploratory process improvement of the quality and quantity of fishery resources in the study area.
\end{abstract}

Keywords: Morocco, Mehdia, Fisheries resources, Maritime fishing, Sustainability.

\section{Introduction}

De par sa position géographique favorable, le Maroc dispose de deux façades maritimes d'environ $3500 \mathrm{~km}$ de côtes, soutenues par une Zone Economique Exclusive (ZEE) de 200 miles marins en Atlantique. Ces atouts, conjugués à l'existence d'une zone d'upwelling (Orbi, 1998), considérée parmi les plus importantes au monde, font de la côte marocaine l'une des zones les plus poissonneuses, avec un potentiel de production annuelle qui dépasse 1,5 millions de tonnes de poisson (Doukkali et Kamil, 2018).

Le secteur de la pêche en effet contribue à hauteur de 2 à $3 \%$ du PIB (Azaguagh, et Driouchi, 2018) et emploie environ 660.000 personnes, de façon directe ou indirecte (Banque mondiale, 2013), tout en assurant des 
sources de revenus pour environ 3 millions de personnes (DPM, 2014). En 2015, la production halieutique nationale a dépassé 1,35 millions de tonnes (d'une valeur de 10,8 milliards de dirhams), composée principalement de petits pélagiques ( $84 \%$ en volume et $25 \%$ en valeur). Grâce à cette production, le Maroc se place au 17ème rang des pays producteurs (pêche de capture) et premier sur le continent africain (FAO, 2016). Cette production est principalement générée par une flotte de pêche composée de navires côtiers et artisanaux (56\% en volume; $54 \%$ en valeur) et de navires hauturiers (34\% en volume ; 44\% en valeur). Cependant, malgré l'importance de la production halieutique nationale, la consommation de produits de la pêche par les marocains reste limitée à des niveaux inférieurs à la moyenne mondiale (13,3 $\mathrm{kg} /$ habitant contre $19,3 \mathrm{~kg} /$ habitant à l'échelle mondiale en 2012). Le Maroc se fixe pour objectif stratégique, dans le cadre du Plan « Halieutis », de porter cette moyenne à $16 \mathrm{~kg} /$ habitant / an vers 2020 (Doukkali et Kamil, 2018). La stratégie s'articule autour de trois axes majeurs : la durabilité de la ressource, la performance et la compétitivité du secteur (Saad et Maria, 2017).

Toutefois, le secteur des pêches maritimes affronte un certain nombre de défis dont les principaux sont liés à la fragilité de la ressource, à la variabilité des conditions hydroclimatiques, à un effort de pêche excessif et aux barrières de la compétitivité et de la qualité. La quasi-totalité des stocks halieutiques sont pleinement exploités voire surexploités à cause d'un effort de pêche excessif dépassant les capacités de reproduction biologiques de la ressource (Abdellah, 2006). C'est le cas notamment de Mehdia qui malgré qu'elle s'ouvre sur l'océan atlantique avec un littoral long $60 \mathrm{~km}$, et renferme des potentialités importantes dans le secteur de pêche (espèces pélagiques, demersales, benthiques), ne profité que peu de sa situation géographique (Motib, 2019).

L'objectif de cette étude vise dans un premier temps, à établir un diagnostic de l'état des ressources halieutiques à Mehdia par le biais d'un inventaire et d'une évaluation du processus de dégradation ainsi que les dommages qu'elles ont subis. Ensuite, il faudrait dégager une stratégie de gestion qui sera adoptée par les acteurs présents, susceptibles d'être insérer dans une orientation non seulement écologique mais socialement durable.

Pour atteindre ces objectifs, la méthodologie suivante a été retenue.

\section{Matériel et méthodes}

\section{Méthodologie du travail}

\section{Capitalisation des informations existantes : bibliographie, données}

Cette étape, consiste essentiellement en la collecte de deux types d'information. La première concerne classiquement la consultation des travaux universitaires (thèses, mémoires et articles scientifiques, livres, communications à des colloques et symposiums, etc.) et la deuxième concerne 
les documents institutionnels (documents de Plan, de Programme, de Projet, etc.). Certaines informations sont également tirées de supports électroniques (Internet). La recherche documentaire permet de faire le point sur l'état des connaissances concernant les écosystèmes côtiers, la gouvernance des ressources halieutiques nationales, les méthodes et outils de détermination d'indicateurs, les théories et les expériences en matière de gestion intégrée des ressources halieutiques. Les informations trouvées dans la littérature servent également d'illustration dans l'interprétation de certains aspects de l'étude.

\section{Les données du terrain et d'entretien}

L'objectif de cette étape est de confronter le cadre théorique d'appréhension et d'analyse de la problématique de la durabilité des ressources halieutiques à une expérience empirique. À ce titre, en vue de développer le propos théorique. Les informations existantes, sont complétées par des enquêtes et des sondages d'opinion sur le terrain.

\section{Les ressources halieutiques à Mehdia: état des lieux et diagnostic \\ 1.1 Des ressources halieutiques diversifiées}

La zone d'étude s'ouvre sur l'océan atlantique avec un littoral long de $60 \mathrm{~km}$ se trouve entièrement dans la province de Kenitra. De ce fait, la zone renferme des potentialités importantes dans le secteur de la pêche (Figure 1), et dispose d'une flotte pour la pêche côtière, artisanale.

La distribution spatiale d'espèces de pêche est fortement liée aux conditions hydro climatiques du milieu et à la nature du fond marin (Med Ocean, 2009).

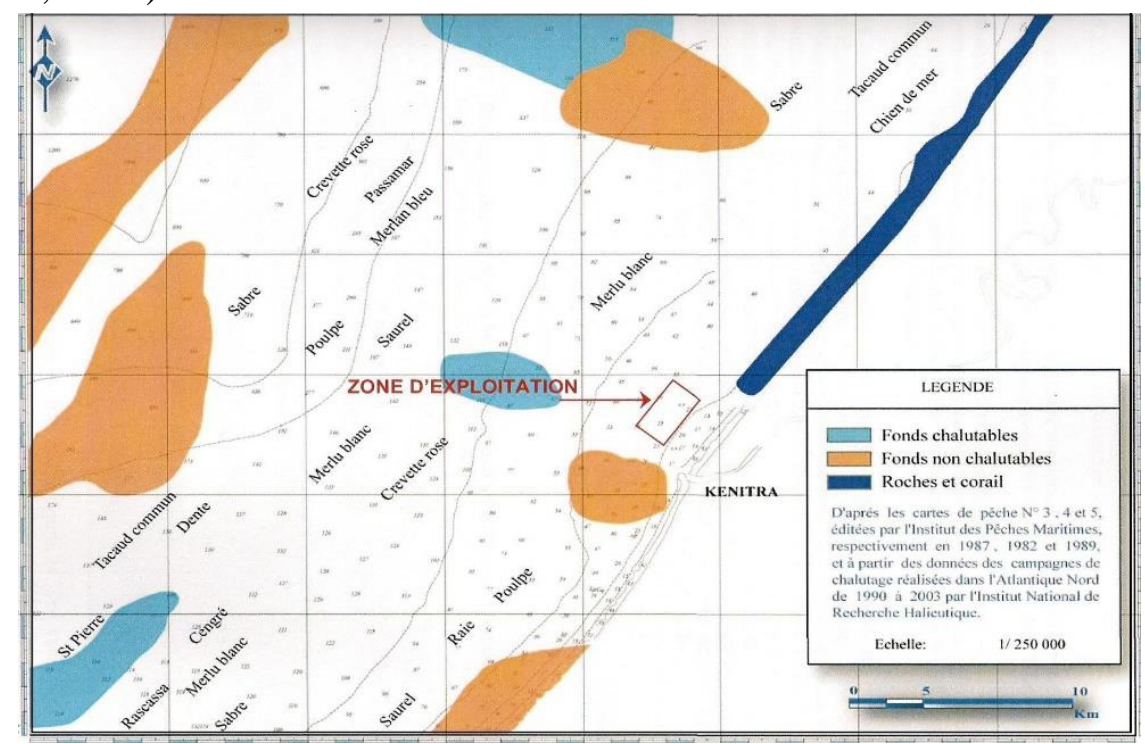

Figure 9: carte des pêches dans la zone de Kenitra (Mehdia), (source INRH) 
Au niveau de Mehdia, les ressources halieutiques sont exploitées par une flotte de pêche nationale, constituée :

- d'une flottille de pêche côtière, non spécialisée, qui opère près de la côte avec des unités de pêche traditionnelles et qui est caractérisée par une diversité de métiers ;

- d'une flottille de pêche hauturière à très large rayon d'action, qui cible essentiellement la crevette rose du large ;

- d'une flottille de pêche artisanale, qui opère au niveau de la bande côtière à l'aide de barques à moteur et qui cible selon la saison et la zone de pêche, soit les poissons blancs, soit les grands crustacés.

Ces ressources sont constituées essentiellement :

\section{Les ressources pélagiques}

Les stocks de pélagiques, sont composés comme suit :

Les stocks des petits pélagiques sont représentés essentiellement par la sardine ((Sardina pilchardus), qui occupe le premier rang en termes de débarquement, suivi de l'anchois (Engraulis encrasicolus), du maquereau (Scomber colias), du chinchard (Trachurus trachurus et Trachurus trecae) et de la sardinelle Sardinella aurita et Sardinella maderensis) (Hakkou, 2012). Ils représentent sur le plan quantitatif les principales ressources exploitées avec $21 \%$ environ de captures.

Toutefois la disponibilité et l'abondance de ces espèces sont étroitement liées à celle des facteurs environnementaux, en particulier l'intensité et la variabilité saisonnière ou interannuelle des upwellings. En outre, ces espèces passent la majeure partie sinon la quasi-totalité de leur phase adulte en surface ou en pleine eau, totalement libres à l'égard du fond et indépendantes de la nature du substrat. Elles sont caractérisées par des migrants horizontaux et verticaux importantes (Med Ocean, 2009).

La sardine prend le large au printemps, formant des petits bancs dispersés. Vers la fin du printemps et jusqu'à l'automne, elle se concentre plus près de la côte et forme des bancs denses.

Les stocks des grands pélagiques sont constitués d'espèces appartenant à la famille des Thonidés. Ces derniers sont des espèces hautement migratrices qui entreprennent de grands déplacements dans leur zone de distribution, parcourant souvent des milliers de kilomètres à l'intérieur de leurs aires de distribution géographique. Les principales espèces de Thonidés sont Thon rouge, Espadon, la Bonite, l'Auxide, la Palomette et la Thonine commune (Benmohammadi et Labraimi, 2011). Ces espèces effectuent des migrations, déterminées par des impératifs de reproduction et d'alimentation, sous le contrôle de facteurs hydrologiques et physicochimiques qui conditionnent l'habitat de ces espèces aux différents stades de vie, saisonnièrement et à plus long terme. Parmi les facteurs connus, il y a la 
température de surface, la profondeur de la thermocline, la salinité et le taux d'oxygène dissous. il faut savoir aussi que le maximum des productions de la pêcherie thonière se situe durant ces périodes de migration.

\section{Les espèces démersales}

Selon la nature du fond marin, les ressources démersales peuplant cette zone peuvent être classées en trois grands groupes :

- Les espèces du fond sableux comme les merluchons, les crevettes, les pageots, les soles, les langues, le turbot, les loups et les mulets ;

- Les espèces du fond vaseux et sablo-vaseux telles que les merlus, les crevettes, la langoustine, les grandes soles, les baudroies, les raies, le tacaud et le merlan bleu ;

- Les espèces des fonds durs et rocheux telles que les grands merlus, les ombrines, le congre, les pagres, les sars, les dentés, les murènes, les grandins, les homards, et les langoustes.

Les zones rocheuses occupent une grande partie du plateau continentale et abritent un potentiel important d'espèces nobles (Hakkou, 2012).

Les principaux stocks démersaux peuplant les fonds meubles (sableux, vaseux et sablo-vaseux) sont représentés par plus de deux cents espèces appartenant principalement aux crustacés, aux merluccidae, aux céphalopodes, aux sparides, aux scorpaenidae, aux marcroudae, aux squalidae, aux sparidae, aux scorpanenidae, aux Gadidae, etc. (Benmohammadi et Labraimi, 2011). Ces espèces représentant une part importante des débarquements de pêche au niveau du port de Mehdia, notamment le merlu commun et la crevette rose du large (Hakkou, 2012).

\section{Les espèces benthiques}

La macrofaune benthique infralittorale de l'estuaire de Sebou est dominée essentiellement par les Mollusques (Tab. 1) suivis de Crustacées puis des Annélides Polychètes (Mergaoui, 2003 et Zine 2005).

Tableau 3: structure taxonomique de la faune benthique infralittorale de l'estuaire de Sebou (source : Mergaoui et al.., 2003, Zine 2005)

\begin{tabular}{|c|c|c|}
\hline Groupes zoologiques & \multicolumn{2}{|c|}{ Nombre d'espèces } \\
\hline & Mergaoui et al., 2003 & Zine, 2005 \\
Mollusques & 30 & $\mathbf{6 2}$ \\
Crustacés & 14 & $\mathbf{2 0}$ \\
Annélides Polychètes & 7 & $\mathbf{1 6}$ \\
Cnidaires & 1 & $\mathbf{1}$ \\
Echinodermes & 1 & $\mathbf{1}$ \\
Poissons & 1 & $\mathbf{2}$ \\
Némertiens & 1 & $\mathbf{2}$ \\
Echiurien & 1 & $\mathbf{1}$ \\
\hline Total & $\mathbf{5 6}$ & $\mathbf{1 0 5}$ \\
\hline
\end{tabular}


L'effort de la pêche concernant les petits pélagiques au niveau des ports de la zone atlantique Nord dont Mehdia fait partie, ont été débarqués par 727 unités actives en 2017 dont 201 senneurs, 312 barques, 174 palangries. (INRH, 2017)

Un total d'environ 17526 sorties a été réalisé à hauteur de $60 \%$ pour les sonneurs côtiers, de $24 \%$ par les chalutiers côtiers. Les barques et les palangriers côtiers ont respectivement contribué avec 14\% et 2\%. Les 212 senneurs côtiers ont opéré dans les 7 ports de la zone (Larache, Mehdia, Mohammedia, Casablanca, El Jadida et Jorf lasfer) en réalisant environ 10980 sorties avec apports. Le port de Mehdia prédomine avec 25\% de sorties en mer, suivi du port en dernier avec $6 \%$ des sorties (figure 2).

En termes d'effectif d'unités de pêche, le port de Mehdia occupe la première position avec 21\%, suivi des ports de Larache (18\%), de Mohammedia (14\%), et le port de Tanger (8\%). (INRH, 2017).

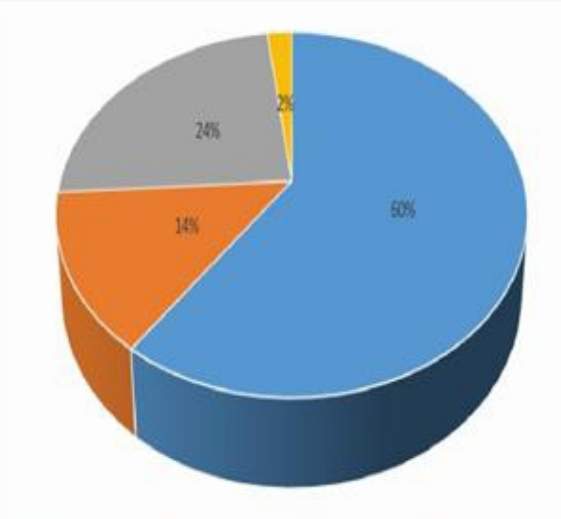

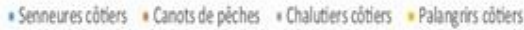

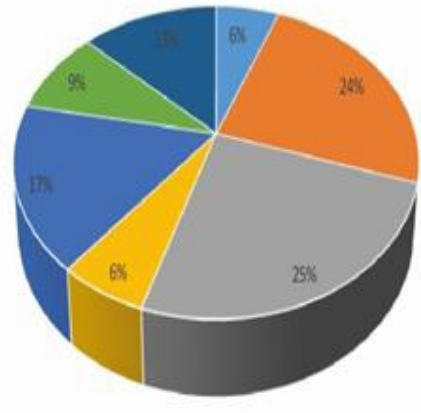

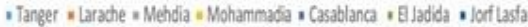

Figure 2 : contribution à l'effort de pêche déployé, en 2017, pour la capture des petits pélagiques dans la zone nord par flotte (à gauche) et par port (à droite) ( Source : INRH 2017).

Cette zone montre aussi, la proximité de plusieurs madragues qui ciblent le thon rouge, les thonidés et l'espadon. D'après la délégation des pêches maritimes de Mehdia-kenitra, le secteur des pêches maritimes emploie (directement et indirectement) plus de 10000 personnes entre les marins embarquant, les mareyeurs et commerçants, travailleurs de chantier naval, ouvriers des unités de transformation des produits de la pêche, etc. (Benmohammadi et Labraimi, 2011).

Le secteur de la pêche participe également à l'alimentation d'une activité industrielle constituée de 18 unités de transformation et de conditionnement. Ces unités se livrent généralement à des activités de semiconserve (salaison et marinade), produits frais, congélation, décorticage d'oursin, cuisson et la transformation des Algues marines. Généralement, la 
capacité de production de ces unités $(22 \%)$ reste très faible par rapport à la capacité maximale de ces unités. Ceci explique en grande partie par l'irrégularité des opérations d'approvisionnement qui reste étroitement tributaire des conditions de travail de la flotte de pêche à partir de ce port )S.D.A.U, 2014).

Tableau 4 : principales activités et capacités de production et du stockage des unités d'industries de pêche au port de Mehdia, Source : SDAU, 2014)

\begin{tabular}{|c|c|c|c|c|c|c|c|c|}
\hline $\begin{array}{c}\text { Unités } \\
\text { agrées }\end{array}$ & \multicolumn{3}{|c|}{$\begin{array}{c}\text { Capacité de production par } \\
\text { jour T/J }\end{array}$} & \multicolumn{3}{c|}{ Capacité de stockage } & \multicolumn{2}{c|}{ Main d'œuvre } \\
\hline & Actuelle & Max & d'utilisation & Actuelle & Max & d'utilisation & Saisonnier & Permanant \\
\hline 18 & 125 & 555 & $22 \%$ & 2727 & 6450 & $42 \%$ & 1585 & 690 \\
\hline
\end{tabular}

En revanche, la construction du futur port atlantique dans la région du Kenitra sur la façade atlantique rendra cette zone beaucoup plus attractive, favorisant ainsi un flux de navires de pêche côtière provenant d'entre ports. Instituée dans le cadre du plan portuaire nationale à l'horizon de 2030, cette plateforme portuaire desservira la région centre et également nord-ouest pour les trafics conventionnels et vrac et notamment les flux issus ou à destination du Gharb, du Saiss et du loukous, des zones franches comme Atlantic free zone.

Ce projet touchera également les activités de pêches maritimes. Là encore, il est fort probable que le niveau de performance de l'activité de pêche soit plus que doublé à l'horizon de la mise en service de nouveau port et ce en raison de :

-l'amélioration des conditions de sortie et d'entrée en mer permettant ainsi la progression des captures des produits de la pêche débarquée par la flotte active.

- l'amélioration de la sécurité de la navigation (SDAU,2014).

\subsection{Diminution des ressources halieutiques à Mehdia}

L'état actuel des ressources halieutiques à Mehdia enregistre, une forte baisse des stocks et des prises, réduction de la qualité des ressources halieutiques, disparition graduelle de certaines ressources halieutiques (anchois, crevettes roses), et pollution de la plage et du milieu marin (Motib, 2019). Ces observations scientifiques sont confirmées par les pêcheurs euxmêmes qui révèlent que la plupart des stocks sont très exploités, voire surexploités. Les causes principales des pressions sur ces ressources sont :

\section{Effets du changement climatique}

Dans le cadre d'une étude comparative globale, (Allison et al., 2009) rapportent que parmi 133 pays, le Maroc est situé au $11^{\text {ième }}$ rang des pays les plus vulnérables aux changements climatiques dans le secteur de la pêche. 
Selon ces mêmes auteurs, les résultats de l'analyse montrent que le Maroc est hautement vulnérable, avec un niveau de capacité d'adaptation faible. Cette vulnérabilité au changement climatique du secteur de la pêche dépend à la fois de l'exposition au risque, de la sensibilité et de la capacité d'adaptation.

Il se dégage des études météorologiques récentes que le Maroc, de par sa situation géographique sensible, connaît des contrastes climatiques régionaux marqués par une forte variabilité des précipitations et une irrégularité des traits climatiques (Banque mondiale, 2013). Le Maroc dispose de deux façades maritimes sur l'Océan Atlantique et la Mer Méditerranée, s'étendant sur une longueur de plus de 3,500 Km. Les conditions hydroclimatiques qui caractérisent les zones de l'Atlantique Sud font de la côte marocaine l'une des plus poissonneuses du monde. Or il est prévu qu'à l'horizon de 2020, le pays connaisse un réchauffement de l'ordre de 0.7 à $1 \%$ (Banque mondiale, 2013).

Au-delà de ces données, des informations sont requises auprès des pêcheurs (117 pêcheurs enquêtés y compris le président de la confédération des pêches côtiers) pour évaluer leur perception des impacts déjà ressentis du changement climatique sur le milieu littoral atlantique (y inclure Mehdia), marin, et sur les ressources halieutiques. Leur perception du changement climatique est liée aux contraintes de la météo, à l'augmentation des températures des eaux, au changement des courants marins et à la disponibilité de la ressource halieutique.

La météo est le premier facteur limitant la pêche au Maroc et à Mehdia, car les pêcheurs n'osent plus sortir en mer quand la mer est agitée, les vents sont forts et que la hauteur des vagues dépasse deux mètres. C'est ainsi que le «mauvais temps » a un impact négatif sur la productivité et la rentabilité de la pêche. Compte tenu de la fréquence et de la durée des périodes de mauvais temps, les pêcheurs sont dans l'obligation de réduire leur nombre de sorties en mer.

Selon le président de la confédération nationale de la Pêche Côtière «CNPC » "Larbi Mhidi", la température est un facteur clé dans la vie du poisson, sa croissance, sa reproduction et sa survie ; la hausse des températures des eaux à Mehdia a entrainé le déplacement des stocks des petits pélagiques vers d'autres zone notamment Safi, Essaouira et le Sud. En plus, de l'avis des pêcheurs, certaines espèces existaient dans la zone de Mehdia aujourd'hui, à cause du changement climatique, a définitivement quitté la zone, notamment l'anchois. Par ailleurs, le changement des courants marins, a entraîné la détérioration des filets de pêche. Ces changements ont sans aucun doute une influence plus importante sur les pêcheurs des petits pélagiques et chalutiers que certains d'eux, ont choisi de vendre leurs bateaux et d'autres de migrer vers d'autres régions. 


\section{Effets de dragage sur la biodiversité du milieu et ressources halieutiques}

Le dragage des sédiments marins est devenu une pratique assez courante dans de nombreux pays depuis plusieurs années. Il est devenu pour certaines zones la principale source d'agrégats pour les besoins du béton, des travaux publics et les travaux de rechargement des plages (Cooper et al. 2007a). Toutefois, l'extraction de sable en mer peut avoir des impacts néfastes sur l'environnement marin, non seulement sur le plan morpho-dynamique de la région, mais aussi sur les écosystèmes locaux, et ce n'est souvent pas limité à la zone d'excavation (Kubrick et al. 2007 ; Van Rijn et al. 2005).

L'activité de dragage du sable au Maroc est d'âge relativement récent. Elle est apparue au début du 20ème siècle (Hakkou et al, 2015), avec l'établissement de ports sur le littoral. Il s'effectue dès l'installation des aires portuaires et se continue pour entretenir et/ou approfondir les chenaux et les bassins pour permettre l'accès des navires aux installations portuaires (Benmohammadi et Labraimi, 2011). Les zones de production de sables marins se situaient essentiellement sur le proche plateau continental de la façade atlantique marocaine. En 2012, on comptait trois plates-formes de traitement et de commercialisation de sables dragués : celles de Mehdia, d'Azemmour et de Larache, pour une production annuelle totale de 3 millions $\mathrm{m}^{3}$. Par ailleurs, l'importance du gisement sableux du plateau continental marocain a incité les opérateurs à multiplier leur production (Hakkou et al, 2015). On traitera dans ce qui suit l'impact des dragages des fonds littoraux à Mehdia, en se limitant aux conséquences de cette exploitation sur les ressources halieutiques.

La zone draguée à Mehdia s'étend sur $6 \mathrm{~km}^{2}$, par des profondeurs allant de $-6 \mathrm{~m}$ à $-20 \mathrm{~m}$ mais nos observations du déplacement de la drague montrent qu'elle ne va guère au-delà de la courbe de -12 m (DPDPM, 2011). L'action de ces dragues, et le prélèvement de la partie superficielle du fond, entraînent des effets directs et indirects sur l'environnement marin littoral (Benmohammadi et Labraimi, 2011), (figure 3).

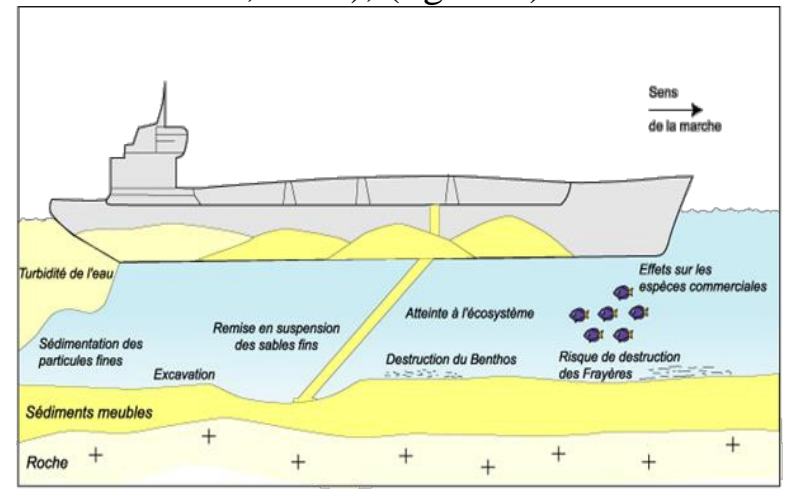

Figure 3 : Synthèse schématique simplifiée des Impacts de l'extraction des granulats marins (IFREMER) 
En ce qui concerne l'effet direct, lors du fonctionnement des dragues, il y'a aspiration d'un mélange d'eau et de sédiment, et d'organismes vivants hôtes de ces sédiments ou se trouvant sur le trajet de l'élinde, par la "tête d'élinde" qui repose sur le fond (largeur $<1 \mathrm{~m}$ ) et qui fouille le substrat meuble sur environ $0,20 \mathrm{~m}$. Il se crée ainsi des sillons d'exploitation. Inévitable, la ponction d'organismes vivants (prélèvement d'individus d'intérêt halieutique) par dragage conduira à appauvrir le peuplement au niveau non seulement du secteur dragué mais bien au-delà. Il faut s'attendre à une réduction notable des densités, de la richesse spécifique et de la biomasse (Benmohammadi et Labraimi, 2011). Sur le fond marin on trouve des zones de ponte pour plusieurs espèces marines et quand on drague les sédiments marins, ces espèces sont automatiquement draguées avec les sédiments et par voie de conséquence la vie poissonneuse est affectée (Benmohammadi, 2011).

Par son augmentation de la température des eaux, de la turbidité et de la redéposition de particules fines (notamment celles issues de « surverse », évacuation en surface de l'eau de décantation des matériaux extraits), les paramètres écologiques sont totalement altérés et affectent directement la flore et la faune qui y vit (« le benthos ») ; La turbidité réduit notamment la pénétration de la lumière, et la photosynthèse; les particules fines peuvent recouvrir le fond et causent la mortalité des espèces qui y vivent; enfin, les organismes vivant sur et dans le fond sont aspirés et donc détruits(Benmohammadi et Labraimi, 2011).

Il y'a aussi des effets à long terme qui peuvent toucher les espèces pêchées et les pêcheries associées, la biodiversité et d'autres intérêts socioéconomiques : disparition du benthos, perturbation de certains écosystèmes qui dépendent étroitement des fonds sableux pour leur reproduction (frayères) ou la nourriture des jeunes pendant certaines phases de leur vie (nourriceries). Ces effets peuvent toucher des espèces exploitées commercialement (voir plus loin les impacts sur la pêche), ou modifier l'équilibre des écosystèmes marins.

Conformément aux dispositions du Plan d'aménagement des pêcheries des crevettes roses, dans certaines zones du pays, la pêche est interdite pendant certaines périodes de l'année. C'est le cas notamment de Mehdia, où les pêcheurs sont appelés à respecter la période du repos biologique (mars et avril), afin de préserver les stocks de la crevette. Or, pendant ce temps, l'activité de dragage continue à Mehdia. Et c'est ce qui explique, selon le président de la CNPC " Larbi Mhid" que les stocks de crevettes, ont connu une baisse très importante. Pour les poissons, près de 60 espèces existaient dans la zone de Mehdia jusqu'aux années 90 Mais, aujourd'hui, à cause du dragage du sable marin, certaines espèces (merlu et crevettes roses) ont définitivement quitté la zone (Motib,2019). 


\section{Effet de Pollution}

Les activités humaines sont responsables de l'introduction dans le milieu marin d'un grand nombre de substances. Selon le PNUE (Programme des Nations Unies pour l'Environnement), 80\% des pollutions marines sont d'origines terrestres et anthropiques (Rachid Amara, 2010). Aux sources industrielles s'ajoutent les effluents domestiques et urbains ainsi que les apports diffus dus aux usages agricoles. L'impact de la pollution des eaux marines est mal mesuré, mais il serait responsable d'importants effets létaux et sublétaux sur les organismes marins (Rachid Amara, 2011).

Il faut noter que l'estuaire de Sebou est situé à proximité de Kenitra et de Mehdia. Ce bassin draine plus que les rejets urbains de la ville de Kenitra, les rejets de la cellulose du Maroc, de la centrale thermique et de la compagnie marocaine du carton et papier de Kenitra. Il connait aussi un forte portuaire avec les ports de pêche à Mehdia et de commerce à Kenitra (Motib, 2019). La partie amont, constitue le réceptacle de diverses activités urbaines, industrielles et agricoles. Ces différentes activités s'accompagnent de nombreux problèmes de pollution qui touchent l'environnement aquatique, et par conséquent le milieu marin, récepteur des eaux continentales drainant des substances polluantes dissoutes ou particulaires (BERRAHO, 2006).

Le suivi des variations temporelles des paramètres physico-chimiques au niveau de l'embouchure a permis d'évaluer l'apport important de nutriments azotés et phosphorés ainsi que la matière en suspension, entrainés par les eaux continentales. L'évaluation des teneurs en métaux lourds dans le sédiment et dans la matière en suspension a montré, qu'avec un maximum 780 ppm, le chrome est l'élément le plus abondant. La présence de cet élément en quantité importante est due aux rejets provenant essentiellement des tanneries. Ceci montre la nécessité de la multiplication des efforts, en vue de limiter la dégradation du milieu marin récepteur, bien que les teneurs observées dans les organismes marins prélevés au niveau de l'embouchure (Mehdia) soient faibles, du fait du pouvoir épurateur du milieu marin. S'agissant des hydrocarbures, il y a lieu de signaler que ces derniers se maintiennent à des concentrations fluctuantes non négligeables, parfois importantes, dénotant l'impact des apports estuariens ainsi que ceux résultant de l'activité du port de Mehdia (BERRAHO, 2006).

\section{Durabilité des ressources halieutiques}

La gestion durable du secteur de la pêche au Maroc et au Mehdia en particulier, s'appuie sur une recherche scientifique efficiente, la mise en place d'un cadre de négociation entre les divers acteurs, un dispositif réglementaire évolutif, des plans d'aménagement efficaces, et une adaptation et une modernisation de l'effort de pêche. 


\section{La mise en place d'une recherche halieutique performante et la formation des professionnels}

L'essor du secteur halieutique au Maroc a suscité, ces dernières décennies, une accentuation de l'effort de recherche pour le suivi de la ressource et la proposition de recommandations pour l'aménagement des pêcheries (cours des comptes, 2018). La compréhension des enjeux de l'exploitation et de l'environnement marin sont déterminants pour la mise en œuvre des mesures de gestion visant la durabilité des pêcheries nationales (INRH, 2018).

Dans cet objectif, Le Maroc a mis en place une recherche halieutique performante comme outil indispensable pour une gestion durable des ressources halieutiques. Il a institué, depuis l'année 2000, un Conseil Supérieur pour la Sauvegarde et l'Exploitation du Patrimoine Halieutique National. Ce dernier constitue un cadre de concertation d'experts scientifiques, de professionnels, de gestionnaires et des autorités (Motib, 2019). En outre, il a créé l'institut National de Recherche Halieutique (INRH), comme acteur de référence de la recherche halieutique, est chargé d'entreprendre toutes activités de recherche, études, actions expérimentales et travaux en mer ou à terre ayant pour objectifs l'aménagement et la rationalisation de la gestion des ressources halieutiques et aquacoles et leur valorisation (DPM, 2014).

A ce sujet, l'INRH a lancé plusieurs stratégies et méthodologies de suivi scientifique sous Halieutis considérant d'une part la diversité des espèces et les modes d'exploitation et d'autre part, les moyens de recherche alloués (INRH, 2018).

En outre, La mise en œuvre d'une gestion durable et concertée des ressources halieutiques et de l'écosystème au Maroc exige un renforcement de capacités humaines par la formation et l'encadrement des professionnels (Motib, 2018). Certes, la formation de l'élément humain occupe une place importante dans la politique du Département de la Pêche Maritime, qui veille à mettre à la disposition des acteurs du secteur les profils disposant des qualifications et compétences nécessaires en matière de conduite, d'exploitation et de maintenance des navires de pêche, ainsi qu'en matière de traitement et valorisation des produits de la mer Ainsi, les actions principales menées durant l'année 2017 ont concerné (DPM, 2017):

- La formation diplômante de ressources humaines nécessaires pour accompagner les différents projets du plan « Halieutis »;

- L'adaptation de l'outil réglementaire, régissant les prérogatives des brevets;

- L'amélioration de la gestion des établissements de formation maritime par l'ancrage de la culture des projets d'établissement; 
- L'appui au secteur en matière de formation continue et d'alphabétisation fonctionnelle

L’élément humain dans la zone étudiée pose problème, aussi bien en termes de formation qu'en termes effectifs. C'est donc une nécessité de renforcer les capacités humaines pour pouvoir initier, gérer et mener à terme des projets liés à la gouvernance des pêches Cela oblige les pouvoirs publics à miser sur la formation de ses cadres et agents afin de les rendre plus compétents, opérationnels et capables d'informer, de sensibiliser et de répondre aux attentes de secteur (Motib,2019).

\section{Un dispositif réglementaire évolutif}

Un ensemble de textes précisant l'étendue du domaine de la pêche maritime et réglementant l'exercice de la pêche ont mis en place au Maroc . En 1919, un code de commerce maritime fut promulgué pour régir l'ensemble des composantes du domaine maritime relatives au commerce, à la navigation et à la pêche maritime en zone française de l'Empire Chérifien (Nejar, 2002).

Le Dahir portant loi $\mathrm{n}^{\circ}$ 1-73-255 du 23 novembre 1973 formant le règlement sur la pêche, a abrogé et remplacé ce code, tout en maintenant certains textes à portée spécifique. (MENFP, 2015). Ce règlement, qui constitue le texte de base actuel, prévoit que l'exercice du droit de pêche est subordonné à l'obtention d'une licence de pêche. Valable pour une année et donnant lieu au paiement d'une taxe (art 2). La pêche à la nage ou pêche sousmarine est assujettie à la délivrance d'une autorisation spéciale (art 4). Par contre, la pêche à pied au moyen de filets fait l'objet d'une simple déclaration auprès du chef de quartier maritime (art 5) (FAO COPEMED, 2002).

Les instruments de pêche et les procédés de pêche font l'objet d'une réglementation plus contraignante. C'est le cas notamment des filets. Par ailleurs, l'exploitation de certaines espèces peut être sujette à des interdictions temporaires (repos biologiques) ou à base de la taille des espèces n'ayant pas atteint des dimensions déterminées tel que prévu par la législation (Nejar, 2002).

Le Dahir de 8 avril 1981 a été promulgué étendant la Zone Economique Exclusive à 200 miles marins. Cette mesure s'inscrit dans un contexte de préservation des richesses marines pour les besoins du développement économique du pays (Nejar, 2002).

Le vide juridique dans certains domaines (estimations de la ressource ou la qualité, salubrité et traçabilité du produit) a été comblé par le recours à des décisions et à des circulaires du Ministre de l'Agriculture et la Pêche Maritime, telles que les arrêtés pour les plans d'aménagement des pêcheries ou la décision de mise en place de la procédure relative à la traçabilité et à la certification des produits de la pêche maritime (cours des comptes, 2018). 
En outre, le Maroc respecte l'ensemble des instruments internationaux en matière de sauvegarde des ressources halieutiques, notamment, le code de conduite pour une pêche responsable de la FAO, les mesures de gestion des thonidés formulées par l'ICCAT, les mesures de gestion de la CGPM et les mesures de protection de la CITES. Pour assurer la pérennité du secteur de pêche à Mehdia, il est nécessaire de réhabiliter les pêcheurs par le biais de cours de formation en textes réglementant ce secteur (Motib, 2019).

\section{Renforcement du Plan d'aménagement}

La planification de l'exploitation des ressources halieutiques repose sur la mise en œuvre des plans d'aménagement et de gestion des pêcheries (Nejar, 2002). La réussite de ses plans d'aménagement des pêcheries maritimes et d'une gestion durable des ressources halieutiques repose sur la disponibilité de données fiables et actualisées sur l'état des ressources. Il est en effet difficile de concevoir une stratégie d'exploitation rationnelle des pêcheries lorsque l'on ignore le potentiel biologique exploitable sur lequel vont s'appliquer les mesures de gestion, car l'effort de pêche doit être ajusté à la taille du stock. Par ailleurs, les pêcheries ne pourront être gérées sans tenir compte des relations entre les ressources et le milieu où elles évoluent et entre les différentes espèces (espèces à valeur économique et espèces sans valeur économiques). (Motib, 2019).

La pêche aux petits pélagiques occupe une place importante dans le secteur des pêches à Mehdia composées essentiellement de sardines, de marquereaux, de chinchards, et de sardinelles (Motib,2019). Le Plan d'aménagement des petits pélagiques vise l'exploitation durable de cette ressource et la maximisation de sa valorisation (DPM,2014). Ce plan fixe notamment :

- Le TAC global par unité d'aménagement ;

- Le tonnage global, le nombre et les caractéristiques des navires autorisés à pêcher les petits pélagiques en tenant compte dans le cas échéant de l'espèce cible ;

- Les périodes et les espaces maritimes dans lesquels la pêche des petits pélagiques est interdite; - Le nombre et les types d'engins autorisés ;

- Le pourcentage des captures accessoires admis ;

- Les modalités de répartition entre les navires autorisés des volumes maximas des captures des petits pélagiques admis ;

- Le ou les ports de débarquement obligatoires, le cas échéant.

\section{Suivi et contrôle pour lutter contre la pêche illicite non déclarée et non réglementée}

La pêche illicite, non déclarée et non réglementée (INN) est un grave problème mondial, l'un des principaux obstacles à la gestion durable des 
pêches. Elle représente une perte importante de revenus (Isolina et Camilla, 2012). Au vu de la gravité des conséquences des pratiques de la pêche illégale sur la pérennité des stocks halieutiques, sur l'écosystème marin et aussi sur la situation socio-économique des opérateurs de la pêche; le souci et la responsabilité nationale vis-à-vis du patrimoine halieutique interpelle le Maroc à mieux gérer, exploiter et conserver de manière durable les ressources halieutiques (DPM, 2014).

Le Plan Halieutis érige la durabilité de la ressource comme un axe majeur et trace parmi ses objectifs une ressource exploitée durablement pour les générations futures. Dans cet objectif, le Maroc a adopté une approche intégrée des dispositifs de suivi, de contrôle et de surveillance des activités de la pêche, des mesures de régulation et un système efficace de traçabilité qui permet de définir l'origine des produits de la pêche au niveau de toutes les étapes de la chaîne de valeur pour lutter contre la pêche illicite non déclarée et non réglementée (DPM,2014). Les autorités à Mehdia utilisent ce système de traçabilité qui assure le contrôle de l'amont à l'aval. (Motib, 2019).

\section{Adaptation et modernisation de la flotte}

Pour contribuer au développement de la pêche, le Maroc veille également à la modernisation de la flotte de pêche, et au renouvellement des navires et des engins de pêche. Il s'agit d'accompagner pour améliorer la sécurité des équipages, l'amélioration des conditions de travail, l'adaptation de la production à la demande pour poursuivre une exploitation durable de la ressource (Motib, 2019). Le programme "Ibhar" initié en 2008, visait le rajeunissement de la flotte, l'optimisation de son rendement, et l'amélioration de la sécurité, de la navigation et des conditions de travail et de vie à bord. Ce programme, prévoyait un investissement global de $5 \mathrm{MMDH}$ dont $1 \mathrm{MMDH}$ du budget de l'Etat (Cour des comptes, 2018).

Dans le contexte de renforcement du processus de réhabilitation et de la modernisation du secteur de la pêche dans le cadre de l'approche intégrée. La chambre des pêches maritimes de l'atlantique nord (CPMAN) a participé à la mise en œuvre du programme Ibhar, qui est un pilier essentiel du programme Halieutis. Ce programme a renforcé les mesures prises par le gouvernement pour aider le secteur à relever une série de défis auxquels sont confrontés les professionnels afin d'améliorer la rentabilité et l'efficacité économique du secteur. Ce programme, qui a suscité une grande satisfaction parmi les professionnels, concerne la mise à niveau des flottes artisanales et côtière ainsi que la modernisation de la flotte côtière (Motib, 2019). La mise à niveau de la flotte artisanale se fait via le pack «Mawarid» qui permet l'équipement des barques par des matériels de sécurité, des caissons isothermes, des moyens de navigation et de surveillance ainsi que des moteurs hors-bords (Motib, 2019). 
Ce pack propose deux options de financement avec une subvention financière à hauteur de $50 \%$ du plafond d'investissement. Le reste peut être un apport personnel ou un crédit. Pack spéciale de navires en bois avec une subvention financière à hauteur de $20 \%$ du plafond d'investissement, et un autre pack relatif aux navires en acier et en fibres de verre avec une subvention financière à hauteur de 30\% (DPM, 2015).

Pour la mise à niveau de la flotte côtière, décliné en trois packs, le pack «ANBAR » qui concerne l'isolation de la cale et de système de réfrigération selon les normes internationales. Le pack «BAHARA » qui vise à équiper les bateaux d'infrastructures sanitaires et sociales. Et le pack « TAJHIZ » permettant l'équipement en moyens de contrôle et de surveillance. En ce qui concerne pack «SAFINA » vise à remplacer les anciennes unités avec de nouvelles (DPM, 2015).

La chambre des pêches maritimes de l'atlantique nord (CPMAN) dans laquelle se situé Mehdia, est considéré comme avant-gardiste, pour le soulèvement de débat sur la nature et le contenu d'un certain nombre des points de programme ayant entrainé des modifications qui ont amélioré l'image du programme lors des journées organisées par la chambre à Casablanca. Les différents protagonistes (les comités locaux relevant de la CPMAN, le comité central de suivi et l'office national des pêches) ont réuni pour étudier les dossiers souhaitant bénéficier du programme Ibhar qui a connu son démarrage officiel et réel par la ratification de 17 dossiers d'un montant financier d'environ dix millions de dirhams. La part de Mehdia été la plus élevée (Motib, 2019):

- $\quad$ Mise à niveau de la flotte de pêche côtière : 5 de 25 .

- $\quad$ Mise à niveau de la flotte de pêche artisanale : 175 de 275.

- Modernisation de la flotte de pêche côtière : 15 de 67.

\section{References:}

1. Abdellah Laouina, 2016: gestion durable des ressources naturelles et de la biodiversité au Maroc, prospective « Maroc 2030 », rapport pour le compte de haute commissariat- au plan, Royaume du Maroc, Mars, p 75.

2. Abdellatif Berraho, 2006 : Évaluation du milieu marin, 25 janvier, $p$ 289.

3. Aicha BENMOHAMMADI, Mustapha LABRAIMI, 2011 : Impacts du dragage d'exploitation sur les fonds littoraux : cas de Mehdia, Conférence Méditerranéenne Côtière et Maritime EDITION 2, TANGER, MAROC, Disponible en ligne - http://www.paralia.fr Available online, p 137.

4. Benmohammadi \& M. Labraimi, 2011:Impacts du dragage des fonds littoraux : Dragage d'exploitation en vue de la commercialisation des 
sables, Défense du Littoral marocain pour un développement durable, Rapport final, avril, p 33-36.

5. Cooper K., Boyd S., Aldridge J. \& Rees H. 2007. Cumulative impacts of aggregate extraction on seabed macro-invertebrate communities in an area off the east coast of the United Kingdom. Journal of sea research, 57: 288-302.

6. Edward H. Allison et Allison,1.Perry, Marie-caroline Badjeck, 2009 : Vulnerability of national economies to the impacts of climate change on fisheries, Fish and Fisheries, june, volume 10, Issue 2, p 173-196.

7. HAKKOU Mounir, 2012 : Contribution à l'étude de la dynamique morphosedimentaire du littoral de bouknadel-kenitra, thèse de doctorat, Université Ibn Tofail, Faculté des Sciences, Kénitra, p 40.

8. 8.HAKKOU Mounir, Aïcha BENMOHAMMADI, Bruno CASTELLE, Xavier BERTIN, Mustapha LABRAIMI, Ahmed EL HASSANI \& Mostapha LAYACHI, 2015 : Perspectives d'optimisation technique de la gouvernance environnementale des activités du dragage du sable marin au Maroc, Bulletin de l'Institut Scientifique, Rabat, Section Sciences de la Terre, 2015, n³7, 00-00, Version numérique ISSN 2458-7184, p 8.

9. Ismail Azaguagh et Ahmed Driouchi, 2019: Gestion des ressources halieutiques au Maroc et modes d'accès : Le modèle des «AntiCommons» et la pêcherie poulpière, Revue Marocaines de Sciences. Agronomiques et Vétérinaires, volume $7 \mathrm{n} \mathrm{1,p} 5$.

10. Ibtissam Motib, 2019 : protection juridique et gestion durable des ressources naturelles au Maroc : cas des communes littorales du Gharb, entre Sidi Taibi et Mnasra (province de kenitra), thèse nationale en Géographie Physique et Environnementale, universite Ibn Tofail, Faculté des lettres et des sciences humaines, Kenitra, p 169.

11. Isolina Boto et Camilla La Peccerella : Lutte contre la pêche illicite, non déclarée et non réglementée (INN) : Impacts et défis pour les pays ACPRessources sur la pêche illicite, non déclarée et non réglementée (INN), une série de réunions sur des questions de développement à Cpue, Septembre, p 4.

12. Kubrick A., Manso F. \& Diesing M. 2007. Morphological evolution of gravel and sand extraction pits, tromper wiek, balticsea. Estuarine, coastal and shelf science, 71, 647-656.

13. Mergaoui, L., Fekhaoui, M., Bouya, D., Gheï, A. \& Stambouli, A., 2003. Qualité des eaux et macrofaune benthique d'un milieu estuarien du Maroc : cas de l'estuaire de Sebou. Bulletin de l'Institut Scientifique, section Sciences de la Vie, 2003, $\mathrm{N}^{\circ}$ 25, 67-75. 
14. Mohammed Rachid Doukkali, Abdelkabir Kamili, 2018: Système marocain de production halieutique et sa dépendance du reste du monde, Research Paper, OCP Policy Center, RP-18/07, p 9.

15. Nada Nejar, 2002 : La politique des pêches au Maroc- Analyse et propositions, Ministère de l'économie, des finances, Département de l'Economie, des Finances et de la Privatisation, juillet, $\mathrm{p} 8$.

16. 4 Orbi, A., (1998). Hydrologie et hydrodynamique des côtes marocaines : milieux paraliques et zones côtières. Expo'98- Lisbonne, OKAD éd: $\mathrm{p} 68$.

17. Philippe cacaud, 2002 : revue de la règlementation relative à la pêche maritime et aux aires protégées dans les pays participants au projet copemed, Mars, p 14.

18. Rachid El Gharbi, 2004 : La mauvaise gestion des ressources halieutiques grève le profit national, le Matin, mise en ligne le 27 décembre, https://lematin.ma/journal/2004.

19. Rachid Amara, 2011 : Impact de la pollution sur les écosystèmes côtiers : exemple de la Manche orientale, la revue électronique en sciences de l'environnement " Vertigo », juillet 2011, https://doi.org/10.4000/vertigo.10990.

20. Rachid Amara, 2010 : Impact de l'anthropisation sur la biodiversité et le fonctionnement des écosystèmes marins. Exemple de la Manchemer du nord, la revue électronique en sciences de l'environnement « Vertigo $\gg, \quad$ octobre, disponible en https://doi.org/10.4000/vertigo.10129.

21. Saad Belghazi et Maria Sarraf, 2017 : Littoral, Le Coût de la Dégradation de l'Environnement au Maroc, environment and natural resources global Practice discussion Paper \#5, Janvier, Groupe de la Banque Mondiale, p 104.

22. Van Rijn L., Soulsby R., Hoekstra P. \& Davies A.G. 2005. Sandpit, sand transport and morphology of offshore mining pits. Aqua publications, The Netherlands, p 156.

23. Zine, N.E., 2005. Etude éco-biologique et dynamique des populations de la palourde (Ruditapes decussatus) et analyse typologique de la faune benthique de milieux paraliques atlantico-méditerranéens du Maroc. Thèse Doctorat d'Etat, Université Moulay Ismail, Meknès, 200p.

24. Banque mondiale, 2013 : Changement climatique et secteur halieutique: Impacts et recommandations, Programme d'Appui Analytique à la Stratégie Changement Climatique du Maroc, Royaume du Maroc, P-ESW 113768 Note de Stratégie n. 3, Décembre, Département du développement durable (MNSSD)Région MoyenOrient et Afrique du Nord, p 3. 
25. Banque mondiale, 2013 : Changement climatique et secteur halieutique: Impacts et recommandations, Royaume du Maroc, NAMA Programme Halieutique-Document Préparatoire, programme d'Appui Analytique à la Stratégie Changement Climatique du Maroc P-ESW 113768, décembre, Département du développement durable (MNSSD $\mathrm{p} 17$.

26. DPM, (2014). Rapport d'activité 2014. Département de la Pêche Maritime relevant du Ministère de l'Agriculture, de la Pêche Maritime, du Développement Rural et des Eaux et Forêts.

27. FAO, (2016). La situation mondiale des pêches et de l'aquaculture 2016. Contribuer à la sécurité alimentaire et à la nutrition de tous. Rome. $224 \mathrm{p}$

28. INRH/DP 2017-Rapport annuel de Etat des stocks et de pêcheries Marocains 2017, Royaume Du Maroc, laboratoires centraux Casablanca, 1 ère Edition, $p 12$.

29. Med Ocean, 2009 : Etude d'impact environnemental, projet dragage et de production de sable marin au large du Sebou, étude d'investigations marines, $\mathrm{p} 39$.

30. Ministère de L'urbanisme et de L'aménagement du Territoire 2014, agence urbain de Kenitra sidi Kacem, SDAU du grand Kenitra, plans d'aménagement, Mission I, diagnostic et orientation, p 296.

31. Ministère de l'Éducation nationale, et de la Formation Professionnelle, 2015: portrait de secteur de pêche maritime au Maroc, octobre, p 27.

32. Ministère de l'Agriculture, de la Pêche Maritime, du Développement Rural et des Eaux et Forêts, Département de la Pêche Maritime, 2017 : Halieutis, rapport d'activité, p 62. 